\title{
Closed Form Expressions for SER and Capacity of Shot Noise Limited MIMO-FSO System Adopting MPPM over Gamma-Gamma Atmospheric Turbulence Channels
}

\author{
Haitham S. Khallaf*and Hossam M. H. Shalaby \\ Egypt-Japan University of Science and Technology (E-JUST), Alexandria, Egypt \\ *haitham.khallaf@ejust.edu.eg, ^shalaby@ieee.org,
}

\begin{abstract}
Closed form expressions for the symbol-error rate (SER) and capacity of shot-noise limited multiple-input multipleoutput free space optical (MIMO/FSO) communication systems, adopting multipulse pulse-position modulation (MPPM) techniques over gamma-gamma channel model, are derived.
\end{abstract}

\section{INTRODUCTION}

Free space optics (FSO) suffers from channel fading during the optical signal transmission due to atmospheric turbulence. These fluctuations cause degradation in system performance. Atmospheric fading can be mitigated through multiple-input multiple-output (MIMO) techniques. The key is to place $M$ lasers and $N$ photodetectors sufficiently far from one another, e.g., $4 \mathrm{~cm}$ for wavelength $1550 \mathrm{~nm}$ and distance $1 \mathrm{~km}$ between transmitter and receiver, to ensure with a high degree of probability that the $M N$ paths are independent [1]. This type of diversity would improve the performance of FSO under strong turbulence channels.

Our aim in this paper is to derive average SER and ergodic capacity formulae for MIMO-FSO systems adopting intensity modulation/direct detection MPPM techniques in gammagamma channels for shot-noise limited receivers. In addition, we use these expressions to study the system performance under strong turbulence for different MIMO system designs.

\section{System AND ChanNEL Models}

We consider a MIMO-FSO communication system with an array of $M$ laser sources all pointed towards a distant array of $N$ photodetectors. We assume that the $M$ laser sources and $N$ photodetectors are placed sufficiently far from one another (the distance between any two elements $>$ correlation distance) such that each of the individual paths from transmitter to receiver is independent. In MPPM techniques $w \in\{1,2, \ldots, Q / 2\}$ pulses are transmitted during a symbol frame of $Q$ time slots. Neglecting the background noise, the detected photon count at photodetector $n$ in time slot $q$ follows a Poisson distribution with mean $\lambda_{o n, n}$ for a signal slot and zero mean for non-signal slots. $\lambda_{o n, n}$ is given by [1]:

$$
\lambda_{o n, n}=\frac{\eta p T}{h f M} \sum_{m=1}^{M} g_{n m}^{2},
$$

where $\eta$ is the photodetector's efficiency, $p$ is the average received optical power in a signal slot, $T$ is the time slot duration, $h$ is Planck's constant, $f$ is the operating frequency, and $g_{n m}^{2}$ is the intensity gain along the path from transmitter $m$ to receiver $n$. We assume that $\left\{g_{n m}\right\}$ are independent and identical distributed random variables.

Several channel models have been assumed to characterize the intensity gain in atmospheric turbulent channels but gamma-gamma can be considered as a general model and is suitable for both strong and weak atmospheric turbulence [2]. Its probability-density function is given by:

$$
\begin{aligned}
& f_{G}\left(g_{n m}\right) \\
& =\frac{2(\alpha \beta)^{\frac{\alpha+\beta}{2}} g_{n m}^{\frac{\alpha+\beta}{2}}-1}{\Gamma(\alpha) \Gamma(\beta)} K_{\alpha-\beta}\left(2 \sqrt{\alpha \beta g_{n m}}\right), g_{n m} \geqslant 0
\end{aligned}
$$

where $\alpha$ and $\beta$ are the scintillation parameters, $\Gamma(\cdot)$ is the gamma function and $K_{c}(\cdot)$ denotes the $c_{t h}$ order modified Bessel function of the second kind. Both $\alpha$ and $\beta$ are dependent on the unitless Rytov variance $\sigma_{R}^{2}=$ $1.23 C_{n}^{2}(2 \pi / \lambda)^{\frac{7}{6}} L^{\frac{11}{6}}$, where $C_{n}^{2}$ is the refractive-index structure parameter, $\lambda$ is the operating wavelength, and $L$ is the distance between the transmitter and receiver.

\section{SyMbOL-ERROR RATE ANALYSIS}

The receiver sums the number of received photons from each detector at each time slot, then it searchs for $w$ largest sums, assuming equal gain combining. A symbol error takes place if $i$ of the $w$ sums are zeros due to photodetectors' efficiency, $i>0$. The SER (for a given path $g_{n m}, S E R_{G}$ ) in that case is given in (3) at the top of next page [1]. The average SER is obtained by averaging $S E R_{G}$ with respect to $g_{n m}$, so we need to calculate $M N$-fold integrations given in (4). Each term in this $M N$-fold integration is identical so it can be rewritten as given in (5). By expressing $K_{v}(\cdot)$ and $\exp (\cdot)$ in terms of the Meijer G function [3], we get (6). Finally, using the integral form for the Meijer G function [3], we get a closed form expression for the SER as given in (7). Similarly, we can get the average ergodic capacity of MIMOFSO system as given in (8). 


$$
\begin{aligned}
& S E R_{G}=\sum_{i=1}^{w} \sum_{l=0}^{w-i}(-1)^{l} \frac{\left(\begin{array}{c}
Q-w+i \\
i
\end{array}\right)-1}{\left(\begin{array}{c}
Q-w+i \\
i
\end{array}\right)}\left(\begin{array}{c}
w \\
i
\end{array}\right)\left(\begin{array}{c}
w-i \\
l
\end{array}\right)\left(e^{\frac{-\eta p T}{h f M} \sum_{n=1}^{N} \sum_{m=1}^{M} g_{n m}^{2}}\right)^{(i+l)} \\
& S E R=\sum_{i=1}^{w} \sum_{l=0}^{w-i}(-1)^{l} \frac{\left(\begin{array}{c}
Q-w+i \\
i
\end{array}\right)-1}{\left(\begin{array}{c}
Q-w+i \\
i
\end{array}\right)}\left(\begin{array}{c}
w \\
i
\end{array}\right)\left(\begin{array}{c}
w-i \\
l
\end{array}\right) \prod_{n=1}^{N} \prod_{m=1}^{M}\left[\int_{0}^{\infty} e^{\frac{-\eta \eta p T}{h f M} g_{n m}^{2}(i+l)} f_{G}\left(g_{n m}\right) d g_{n m}\right] \\
& S E R=\sum_{i=1}^{w} \sum_{l=0}^{w-i}(-1)^{l} \frac{\left(\begin{array}{c}
Q-w+i \\
i
\end{array}\right)-1}{\left(\begin{array}{c}
Q-w+i \\
i
\end{array}\right)}\left(\begin{array}{c}
w \\
i
\end{array}\right)\left(\begin{array}{c}
w-i \\
l
\end{array}\right)\left[\int_{0}^{\infty} e^{\frac{-\eta p T}{h f M} g^{2}(i+l)} f_{G}(g) d g\right]^{M N} \\
& S E R=\sum_{i=1}^{w} \sum_{l=0}^{w-i}(-1)^{l} \frac{\left(\begin{array}{c}
Q-w+i \\
i
\end{array}\right)-1}{\left(\begin{array}{c}
Q-w+i \\
i
\end{array}\right)}\left(\begin{array}{c}
w \\
i
\end{array}\right)\left(\begin{array}{c}
w-i \\
l
\end{array}\right)\left[\int_{0}^{\infty} \frac{(\alpha \beta)^{\frac{\alpha+\beta}{2}} g_{n m}^{\frac{\alpha+\beta}{2}}-1}{\Gamma(\alpha) \Gamma(\beta)} G_{0,2}^{2,0}\left(\alpha \beta g \mid \frac{\alpha-\beta}{2}, \frac{-}{2}+-G_{0,1}^{1,0}\left(\left.\frac{\eta p T(i+l) g^{2}}{h f M}\right|_{0} ^{-}\right) d g\right]^{M N}\right. \\
& S E R=\frac{(2)^{(\alpha+\beta-3) M N}}{\pi^{2 M N}(\Gamma(\alpha) \Gamma(\beta))^{M N}} \sum_{i=1}^{w} \sum_{l=0}^{w-i}(-1)^{l} \frac{\left(\begin{array}{c}
Q-w+i \\
i
\end{array}\right)-1}{\left(\begin{array}{c}
Q-w+i \\
i
\end{array}\right)}\left(\begin{array}{c}
w \\
i
\end{array}\right)\left(\begin{array}{c}
w-i \\
l
\end{array}\right) G_{4,1}^{1,4}\left(\left.\frac{16 \eta p T}{h f M(\alpha \beta)^{2}}(i+l)\right|^{\frac{1-\beta}{2}}, \frac{2-\beta}{2}, \frac{1-\alpha}{2}, \frac{2-\alpha}{2}\right)^{M N} \\
& C=\log _{2}\left(\begin{array}{l}
Q \\
w
\end{array}\right)-\frac{(2)^{(\alpha+\beta-3) M N}}{\pi^{2 M N}(\Gamma(\alpha) \Gamma(\beta))^{M N}} \\
& \times \sum_{i=1}^{w} \sum_{l=0}^{w-i}(-1)^{l} \log _{2}\left(\begin{array}{c}
Q-w+i \\
i
\end{array}\right)\left(\begin{array}{c}
w \\
i
\end{array}\right)\left(\begin{array}{c}
w-i \\
l
\end{array}\right) G_{4,1}^{1,4}\left(\left.\frac{16 \eta p T(i+l)}{h f M(\alpha \beta)^{2}}\right|^{\frac{1-\beta}{2}}, \frac{2-\beta}{2}, \frac{1-\alpha}{2}, \frac{2-\alpha}{2}\right)^{M N} .
\end{aligned}
$$

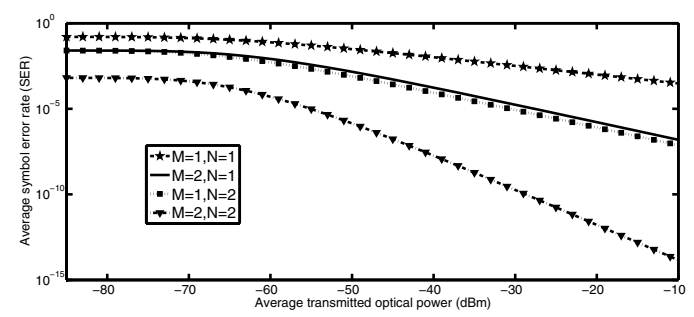

Fig. 1. Average SER versus average transmitted optical power in $\mathrm{dBm}$ for MIMO-FSO system adopting MPPM techniques with $Q=16$ and $w=8$ under strong turbulence channel, $\sigma_{R}=5$, for different MIMO settings.

\section{Numerical Results}

In this section, we use closed form expressions of (7) and (8) to investigate the performance of MIMO-FSO systems adopting MPPM techniques under strong turbulence gammagamma channels. Figures 1 and 2 show both the average SER and ergodic capacity versus average transmitted optical power in $\mathrm{dBm}$ for different MIMO-FSO systems adopting MPPM techniques (with $Q=16$ and $w=8$ ) and different settings of the MIMO system. As shown in the figures, the system performance is improved by increasing the number of lasers as well as the number of receivers. However, for a given $M N$ value, increasing the number of receivers has a more beneficial effect than increasing the number of transmitters due to the increase in overall receiver aperture size.

\section{CONCLUSION}

Closed form expressions for the SER and capacity of shot-noise-limited MIMO-FSO syatems adopting MPPM tech-

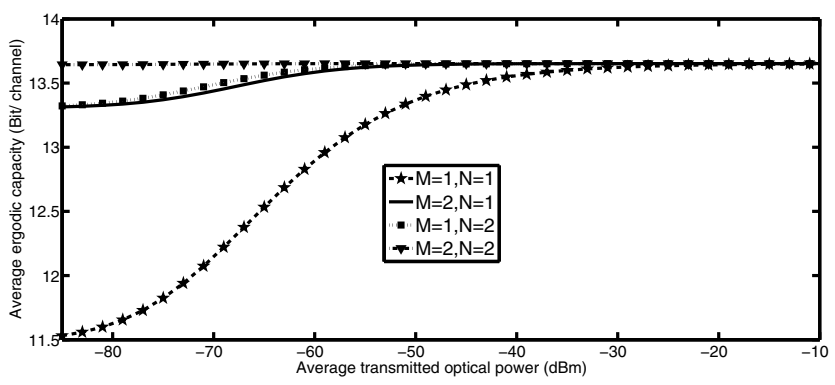

Fig. 2. Average ergodic capacity versus average transmitted optical power in $\mathrm{dBm}$ for MIMO-FSO system adopting MPPM techniques with $Q=16$ and $w=8$ under strong turbulence channel, $\sigma_{R}=5$, for different MIMO settings.

niques is derived. These expressions are used to investigate the system performance under strong turbulence channels. We conclude that increasing number of both transmitters and receivers would improve the system performance. However, increasing number of receivers has more effect on system performance.

\section{REFERENCES}

[1] S. G. Wilson, M. Brandt-Pearce, Q. Cao, and M. Baedke, "Optical repetition mimo transmission with multipulse ppm," IEEE J. Sel. Areas Commun, vol. 23 no. 9, pp. 1901-1909, 2005.

[2] R. L. Al-Habash, M. A. Andrews, and L. C. Phillips, "Mathematical model for the irradiance probability density function of a laser beam propagating through turbulent media," Opt. Eng., vol. 40, no. 8, pp. 1554 1562, Aug. 2001.

[3] Wolfram Function Site. (2013, Feb.). [Online]. Available: http://functions.wolfram.com/ 